\title{
AVALIAÇÃO DO POTENCIAL DE UTILIZAÇÃO DOS DADOS DA SRTM - SHUTTLE RADAR TOPOGRAPHY MISSION POR MEIO DA ANÁLISE DA ESTIMATIVA DE PERDA DE SOLO.
}

\author{
ASSESSMENT OF SRTM - SHUTTLE RADAR TOPOGRAPHY MISSION DATA \\ POTENTIAL USE THROUGH ESTIMATED SOIL LOSS ANALYSIS.
}

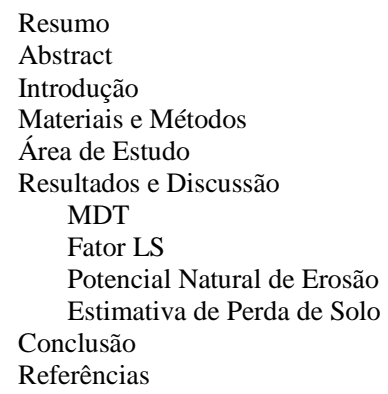

Fernanda Giffoni Fernandes LUZ ${ }^{1}$, Camila Andrade $\mathrm{ABE}^{1}$, Francisco Antônio DUPAS ${ }^{2}$, Júlio César de Souza MATOS ${ }^{1}$, Janine Ameku NEVES ${ }^{1}$

(1) Universidade Federal de Itajubá, Instituto de Recursos Naturais, NEPA - Núcleo de Estudo, Planejamento Ambiental e Geomática. Itajubá, MG. Emails: giffoni.f@gmail.com; camilabe@gmail.com; matosj.c.s.@gmail.com; janine.fct@gmail.com (2) Universidade Federal de Itajubá, Instituto de Recursos Naturais. Itajubá - MG. Email: dupas@unifei.edu.br

RESUMO - A qualidade dos resultados depende da acurácia dos dados disponíveis. Dados topográficos de todo o globo, provenientes da SRTM - Shuttle Radar Topography Mission, são disponibilizados gratuitamente. Se corretos, estes dados constituem recurso poderoso para o planejamento territorial e ambiental. O objetivo deste trabalho foi verificar o grau de semelhança da informação disponibilizada pela SRTM com outra de maior resolução, aplicada ao modelo EUPS - Equação Universal de Perda de Solo para estimar a perda de solo em uma microbacia em São Carlos, São Paulo, Brasil. A acurácia do MDT SRTM foi avaliada por meio do índice KAPPA que, através de uma matriz de erro, mostra o grau de semelhança entre dois mapas. O índice KAPPA revelou que o MDT SRTM possui um nível de precisão considerado razoável (0.6594) para os mapas de estimativa de perda de solo, entretanto os mapas do fator LS, potencial natural de erosão e estimativa de perda de solo possuem níveis de acurácia considerados baixo (0.2584), baixo (0.2582) e moderado (0.4199), respectivamente. Finalmente, os dados fornecidos pela SRTM apresentaram resultados razoáveis, quando multiplicados por dados de maior resolução em cenários de relevo pouco acidentado. Contudo devem ser utilizados com precaução em outras situações.

Palavras-chave: SRTM, avaliação de acurácia, planejamento territorial, estimativa de perda de solo.

\begin{abstract}
Quality results depends on the available data accuracy. Topographic data for the entire globe from SRTM - Shuttle Radar Topography Mission are free and, if correct, they represent a powerful resource for the territorial and environmental planning. However, its use, in models for information systems, must be evaluated in order to assess the information reliability. The aim of this study was to verify the similarity between a soil loss map generated from SRTM DEM and another from a higher resolution. USLE model - Universal Soil Loss Equation was applied with the intention of estimate soil loss rates in a basin on the northeast of São Paulo State in Brazil. Therewith it was possible to assess the performance of SRTM DEM. The similarity was checked using the Kappa index, which shows the degree of similarity between maps through an error matrix. The Kappa index revealed that SRTM DEM has an accuracy level considered reasonable (0.6594), while Kappa values for LS factor (0.2584), natural erosion potential (0.2582) and estimated soil loss (0.4199) accuracy levels are considered low, low and moderate, respectively. Therefore, this study showed that the application of SRTM DEM can offer satisfactory results when multiplied by higher quality data in basins where LS is not a decisive factor in estimated soil loss, but must be used carefully in other conditions.
\end{abstract}

Keywords: SRTM, accuracy assessment, territorial planning, soil loss estimation.

\section{INTRODUÇÃO}

O aumento da disponibilidade de dados espaciais possibilita o surgimento de resultados cada vez mais precisos para modelos ambientais. Com tudo, o uso destes dados deve ser constantemente analisado para que a confiabilidade das informações seja assegurada. Tal fato cria uma demanda relacionada à produção de resultados sobre avaliação da qualidade dos dados disponíveis, através de modelagem ambiental (Li et al., 2013; Korada \&
Rao, 2014; Moradi et al., 2014; Shan et al., 2014; Fluet-Chouinard, et al., 2015).

No Brasil, dados da SRTM - Shuttle Radar Topography Mission, corrigidos pela EMBRAPA - Empresa Brasileira de Pesquisa Agropecuária (Rabus et al, 2003; Miranda, 2005) são disponibilizados gratuitamente e representam um recurso valioso para o planejamento territorial e ambiental. Porém esses dados necessitam de avaliação antes de serem usados com segurança. 
Autores (Vente et al., 2009; Yang et al., 2011; Lin et al., 2013; Small \& Sohn, 2015) demonstraram que, para pequenas bacias, a resolução de $90 \mathrm{~m}$ do modelo digital do terreno MDT fornecido pela SRTM apresenta uma representação insatisfatória dos limites da área de drenagem e que, portanto, precaução é necessária para a escolha e utilização dos dados.

A aplicação da EUPS, com o suporte de um S.I.G. e técnicas geoestatísticas, tem recebido atenção especial de pesquisadores, sendo que diversos autores (Farinasso et al., 2006, Pandey et al., 2007, Zhang et al., 2009, Gericke, 2015) demonstraram relevante potencial na sua utilização como ferramenta de gestão da terra.
Se corretos, os resultados obtidos pela aplicação remota de modelos ambientais realizados a baixos custos representam recurso importante para o planejamento espacial.

Diante disto, o objetivo deste trabalho foi avaliar o desempenho do MDT SRTM por meio de resultados para estimativa de perda de solo. Os valores foram estimados através da EUPS Equação Universal de Perda de Solo (Wischmeier \& Smith, 1978). A avaliação da precisão dos dados foi feita utilizando-se o índice KAPPA (Cohen, 1960) que, por uma matriz de erro, determina a diferença entre dois mapas (Congalton, 1991; Foody, 2009). Como referência, foi utilizada a carta topográfica do em escala 1:50.000.

\section{MATERIAIS E MÉTODOS}

O MDT SRTM é gratuito e está disponível com resolução de 3 arc sec $(\approx 90 \mathrm{~m})$ (Valeriano \& Rossetti, 2008). No Brasil, a Empresa Brasileira de Agropecuária (EMBRAPA) tratou os dados brutos, gerando MDTs corrigidos (Miranda et al., 2005).

Estes dados foram comparados com os dados da carta topográfica em escala 1:50.000. O MDT SRTM e o MDT gerado a partir da carta topográfica foram usados como dados de entrada para o software USLE-2D (Desmet \& Govers, 1996), o qual calcula os fatores comprimento de rampa e declividade - LS da EUPS para uma bacia hidrográfica. Este fator considera a declividade e o comprimento da rampa que determinam a velocidade e o tempo do escoamento e, portanto, influenciam a quantidade de solo erodido.

A sistematização dos fatores que compõem a EUPS foi realizada com dois MDTs, um proveniente da SRTM e outro da carta topográfica (IBGE, 1972). Posteriormente, foi realizada a multiplicação dos fatores para gerar os mapas de predição do Potencial Natural de Erosão - PNE e de Estimativa de Perda do Solo - EPS.

A Equação Universal de Perda de Solo EUPS (Wischmeier \& Smith, 1978) é um modelo multiplicativo simples, no qual a estimativa de perda média anual de solo é dada em tonelada por hectare por ano (t.ha ${ }^{-1} \cdot$ ano $\left.^{-1}\right)$, a equação é composta pelo produto de seis fatores:

$$
A=R * K * L * S * C * P
$$

Onde: $\mathrm{A}=$ predisposição à perda de solo, em t.ha ${ }^{-1}$. nno $^{-1} ; \quad \mathrm{R}=$ fator erosividade da precipitação e da enxurrada, em MJ.mm $\mathrm{mm}^{-1}$.ha ${ }^{1} \cdot \mathrm{h}^{-1} \cdot$ ano $^{-1} ; \mathrm{K}$ = fator erodibilidade do solo, em ton.ha ${ }^{-1} \cdot \mathrm{MJ}^{-1} \cdot \mathrm{mm}^{-1}$; L = fator comprimento da encosta, adimensional; $\mathrm{S}=$ fator grau de declividade, adimensional; $\mathrm{C}=$ fator de cobertura e uso do solo, adimensional; $\mathrm{P}=$ fator de práticas de controle da erosão, adimensional.

$\mathrm{O}$ fator R para a bacia foi dado pelo software NetErosividade que determina valores de erosividade da chuva para regiões de todo o estado de São Paulo (Moreira et al., 2006).

$\mathrm{O}$ fator $\mathrm{K}$ mensura a erodibilidade do solo, ou seja, a capacidade que cada tipo de solo tem de erodir. Estes valores foram obtidos a partir de consulta à literatura (Silva, 2004; Chaves, 1994) e foram relacionados à carta pedológica (Oliveira \& Prado, 1984).

$\mathrm{O}$ fator $\mathrm{C}$ define a influência da cobertura do solo sobre a perda de solo. Os valores de C foram determinados pelo mapeamento da cobertura do solo realizado por Cunha et al. (2011), que o fez a partir de uma composição colorida falsa cor de imagens do satélite ALOS, com resolução de $10 \mathrm{~m}$. Os valores de C, para cada um dos temas do mapa de cobertura do solo, foram encontrados na literatura (Pimenta, 1998; Farinasso et al., 2006; Weill e Sparovek, 2008; Oliveira et al., 2011).

O fator $\mathrm{P}$ trata das práticas conservacionistas adotadas por cada tipo de cultura. Para este trabalho foi considerado o cenário mais conservador, quando não existem práticas 
conservacionistas sendo utilizadas na bacia, portanto, P é 1 para toda a bacia.

Para avaliar a precisão do MDT SRTM foram utilizadas matrizes de erro, que são matrizes quadradas de números estabelecidos em linhas e colunas que expressam os dados dos mapas investigados (mapas hipsométricos, mapas do fator LS, mapas PNE e EPS) (Congalton, 1991). A partir da matriz de erro é possível determinar o índice Kappa - K (Cohen, 1960) - um dos mais utilizados e eficientes coeficientes empregados na determinação da exatidão de dados (Congalton \& Green, 1993; Foody, 2009). O índice Kappa é obtido mediante a adoção de dados de referência para comparação com os dados produzidos e seu valor varia de -1 a 1 , apontando a semelhança entre os dados cruzados, sendo que valores próximos a -1 indicam que os dados são menos parecidos com os dados de referência e valores próximos a 1 indicam que os dados são mais parecidos.

Matematicamente, o índice Kappa é expresso pela Equação:

$$
K=\frac{N \sum_{i=1}^{r} x_{i i}-\sum_{i=1}^{r}\left(x_{i+} * x_{+i}\right)}{N^{2}-\sum_{i=1}^{r}\left(x_{i+} * x_{+i}\right)}
$$

Onde: $\boldsymbol{N}$ é o número total de pixels contemplado pela matriz de erro; $\boldsymbol{x}$ é o elemento da matriz de erro; $\boldsymbol{r}$ é o número de categorias presentes na matriz de erro; xii são os elementos da diagonal principal.

\section{ÁREA DE ESTUDO}

A bacia hidrográfica estudada possui área de 22.270ha (Figura 1). Os valores altimétricos variam de 600 e 1.000 metros. O solo da bacia é predominantemente Neossolo quartzarênico e Latossolo vermelho amarelo (Nishiyama, 1991). A bacia apresenta relevo levemente acidentado, intercalado com elevações de maiores declividades nas Cuestas Basálticas (Zuquette, 1981). A vegetação encontrada na bacia é do tipo cerrado e floresta estacional semidecídua (Tundisi et al., 2007).

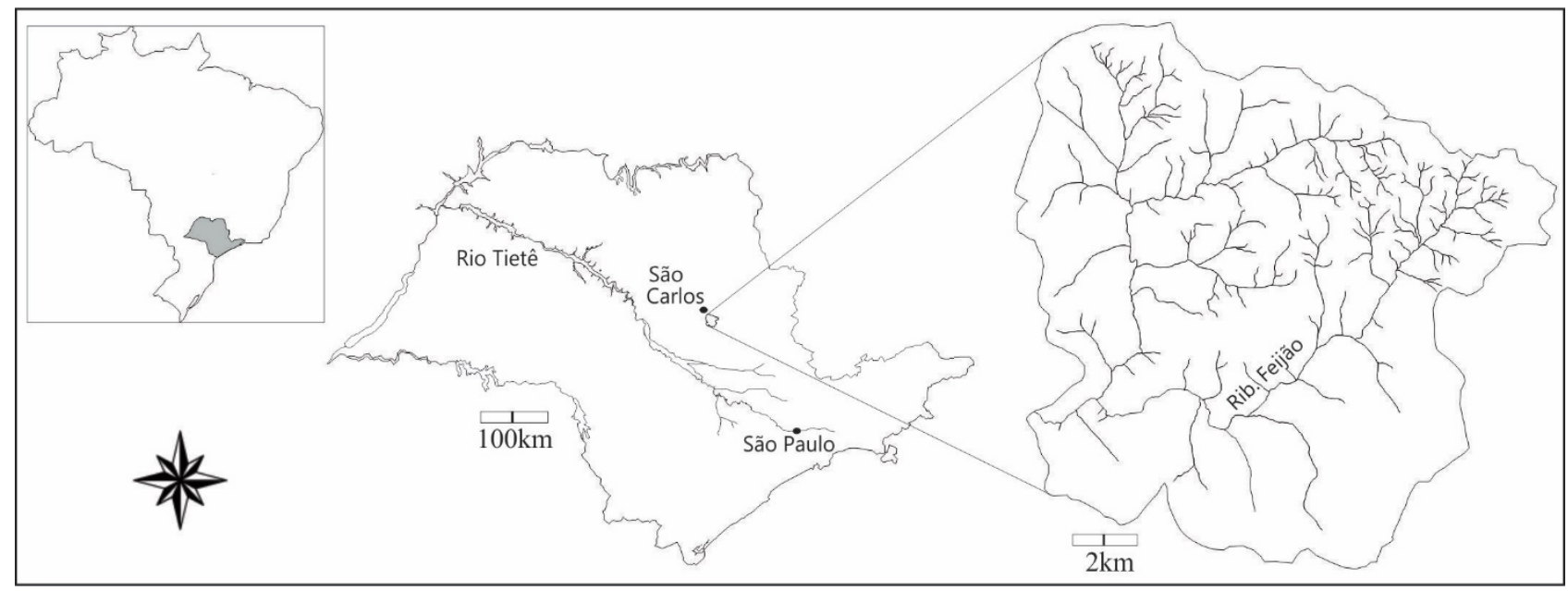

Figura 1- Localização da área de estudo.

\section{RESULTADOS E DISCUSSÃO}

O valor de R para a bacia estudada foi de 7.362 MJ.mm.ha-1.h-1.ano-1. De acordo com Mello et al. (2007), este valor pode ser classificado como alto, o que significa que o fator erosividade exerce grande influência sobre a perda de solo da bacia em questão.

De acordo com o mapa pedológico (Figura 2), o Neossolo quartzarênico ocupa $42 \%$ e o Latossolo vermelho amarelo 33,8\% da bacia. O primeiro está presente nas partes mais baixas das vertentes e o segundo nas partes altas da bacia, onde estão as nascentes dos rios. Juntos, esses solos cobrem 76,2\% da área total da bacia e possuem valor de $K=0,032$ (Tabela 1), de acordo com a classificação proposta por Carvalho (1994, segundo Silva \& Alvares, 2005), são solos com alta vulnerabilidade à erosão. Os valores de $\mathrm{C}$ foram encontrados na literatura (Tabela 2) e estão relacionados ao mapa de uso do solo (Figura 3). 


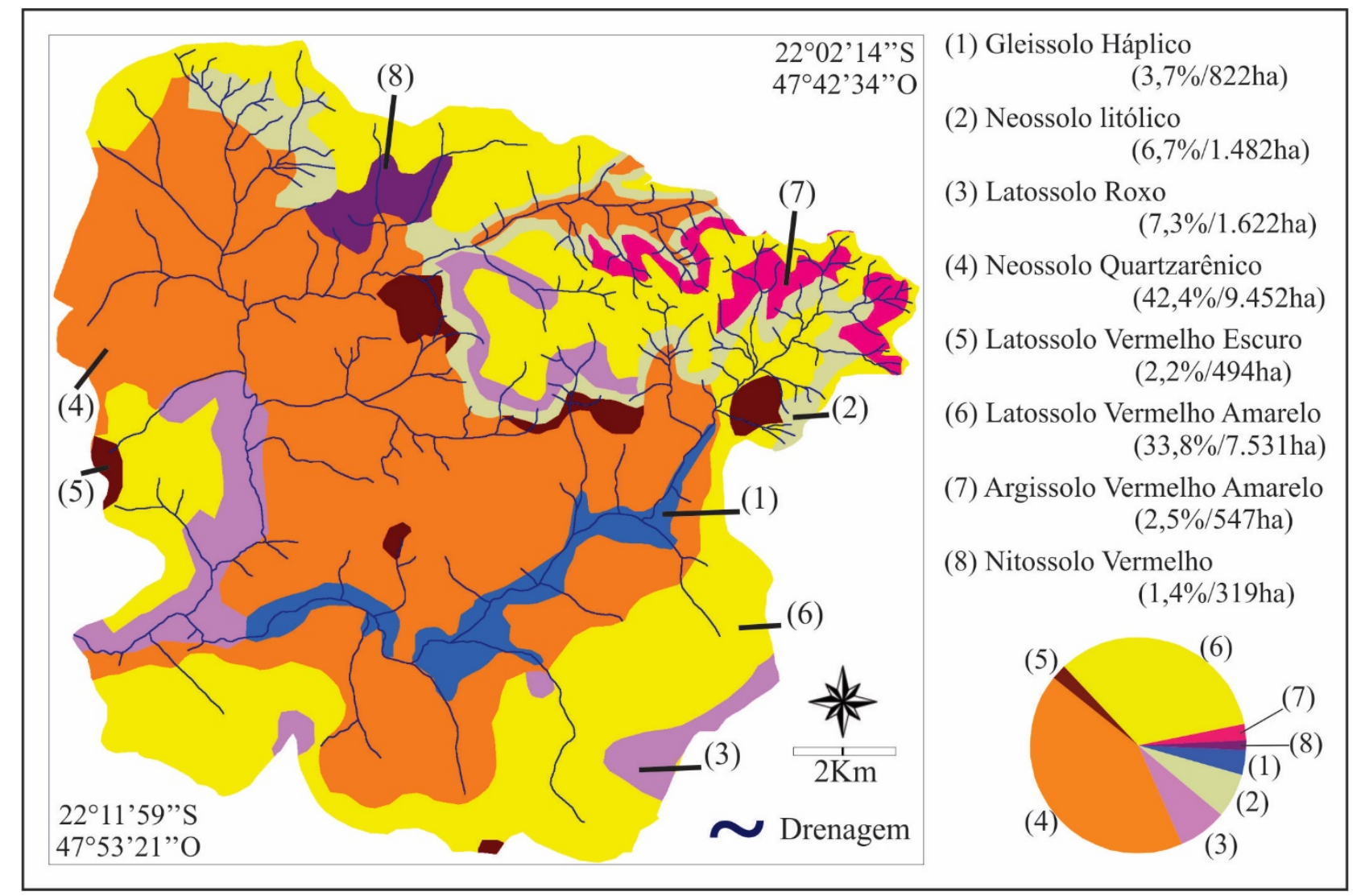

Figura 2 - Mapa pedológico. Fonte: Oliveira \& Prado (1984).

Tabela 1 - Valores médios de K para os 8 tipos de solo da bacia.

\begin{tabular}{|c|c|}
\hline Tipo de solo & $\begin{array}{c}\text { Fator k } \\
\left(\text { t.h.ha }{ }^{-1} \cdot \mathrm{MJ}^{-1} \cdot \mathrm{mm}^{-1}\right) \\
\end{array}$ \\
\hline (1) Gleissolo Háplico & 0,038 \\
\hline (2) Neosolo Litólito & 0,040 \\
\hline (3) Latossolo Roxo & 0,013 \\
\hline (4) Neossolo Quartzarênico & 0,032 \\
\hline (5) Latossolo Vermelho Escuro & 0,014 \\
\hline (6) Latossolo Vermelho Amarelo & 0,032 \\
\hline (7) Argissolo Vermelho Amarelo & 0,040 \\
\hline (8) Nitossolo Vermelho & 0,011 \\
\hline
\end{tabular}

Fonte: (1,2,3,4,5,6,7) Silva, 2004; (8) Chaves, 1994.

Tabela 2 - Fator C.

\begin{tabular}{c|c}
\hline Uso do solo & C \\
\hline (1) Mata ciliar & 0,012 \\
\hline (2) Urbano & 0,001 \\
\hline (3) Cerrado & 0,042 \\
\hline (4) Reflorestamento & 0,001 \\
\hline (5) Solo exposto & 1,000 \\
\hline (6) Pastagem & 0,010 \\
\hline (7) Cana de açúcar & 0,3066 \\
\hline (8) Laranja & 0,200 \\
\hline (9) Água & 0,000 \\
\hline
\end{tabular}

Fonte: (8) Pimenta, 1998; (1, 2, 3, 5) Farinasso et al., 2006; (4) Weill e Sparovek, 2008; (9) Oliveira et al., 2011. 


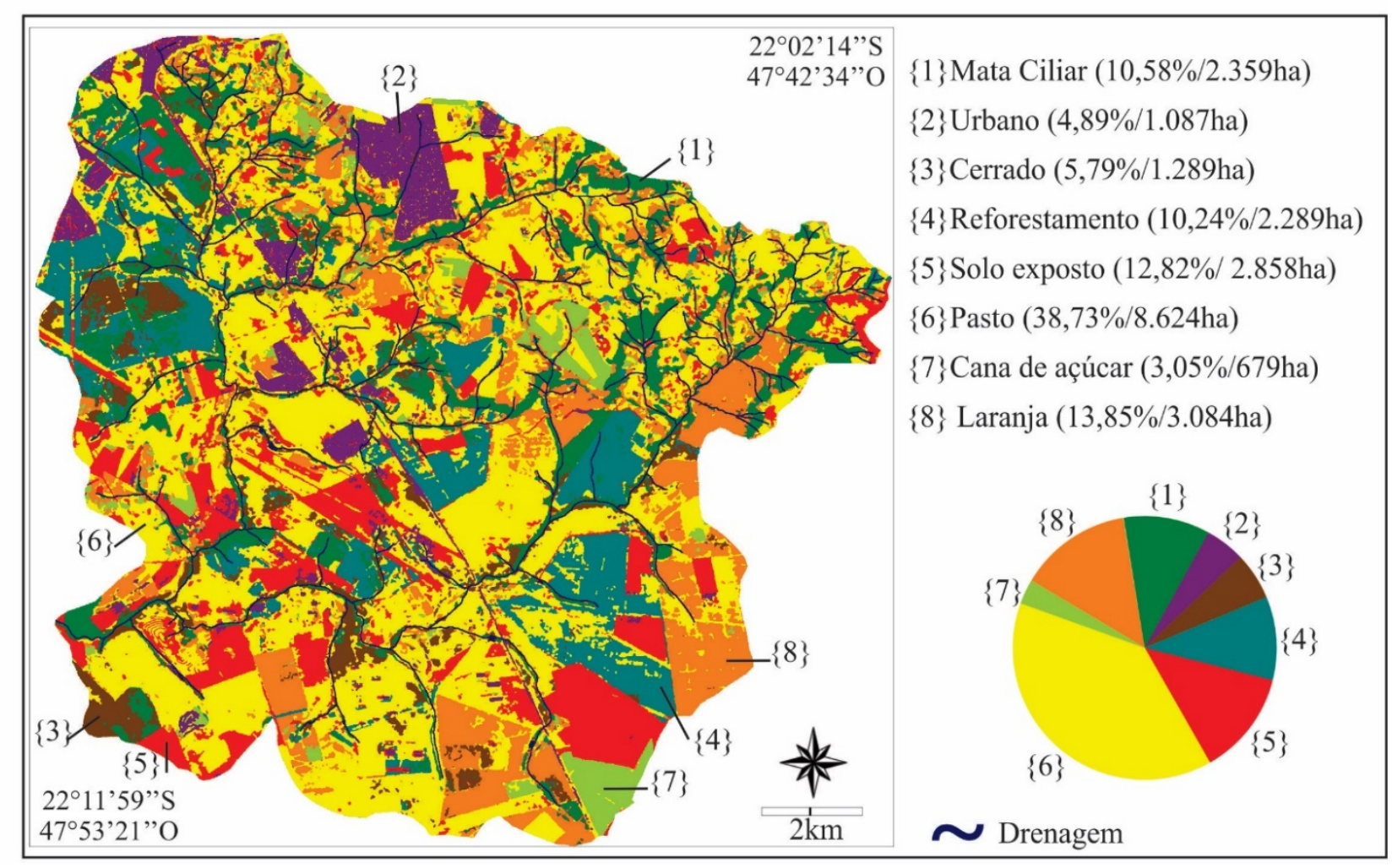

Figura 3 - Mapa de uso do solo. Fonte: Cunha et al., 2011.

Foi assumido o cenário de utilização onde não existem práticas conservacionistas para a atividade agrícola, portanto, o valor de $\mathrm{P}$ foi 1 para toda a bacia.

O mapa de uso do solo mostrou que $38,73 \%$ da bacia são usados como pastagem, seguidos por plantação de laranja (13,85\%) e solo exposto (12,82\%). Cerradão e mata ciliar, coberturas que protegem o solo e os mananciais, ocupam 16,37\% da bacia. Zonas de reflorestamento ocupam 10,24\% e áreas urbanas 4,89\% da bacia. Os dados indicam que $26,61 \%$ da bacia possuem vegetação que protege a terra dos processos erosivos, enquanto que os outros $73,39 \%$ são usados para agricultura e urbanização.

Verificou-se que 4.461ha apresentaram perda de solo maior que $100 \mathrm{t} \mathrm{ha}^{-1}$. ano $\mathrm{o}^{-1}$ e o solo exposto foi a classe com maior frequência, correspondendo a 49,9 da área com maior potencial de perda de solo. Em seguida, estão as culturas de laranja e cana, que somadas correspondem a 39\% das áreas com maior estimativa de perda de terra. O solo exposto está associado às culturas perenes, quando cultivos são retirados e o solo aguarda novo plantio. Os dados sugerem que estas práticas de manejo do solo são inadequadas e resultam em grande perda de recursos naturais e econômicos que impactam o próprio produtor.

\section{MDT}

Resultados apresentados por Rodriguez (2006) indicaram que os dados do MDT SRTM contêm erros absolutos maiores do que os determinados pela missão que coletou os dados. Neste estudo o MDT SRTM (Figura 4) foi comparado com dados de maior resolução.

Através da matriz de erro foi possível identificar que os erros observados estão presentes entre as classes subsequentes dos mapas, sendo que o MDT SRTM apresenta valores de altitude ligeiramente maiores do que o MDT referência. Este fato também foi verificado em trabalhos prévios, como por exemplo Sharma \& Tiwari (2014) e Athmania \& Achour (2014).

Rexer \& Hirt (2014) encontraram evidências de que o MDT SRTM reflete a altura dos objetos presentes na superfície da Terra como vegetação e construções, este fato pode ter sido responsável pelos valores ligeiramente mais altos do MDT SRTM. Ainda pela matriz de erro, nota-se que o total de pixels diferentes do mapa referência corresponde a $27.26 \%$, ou seja, quase um terço dos pixels está alocado em classes diferentes da referência. 


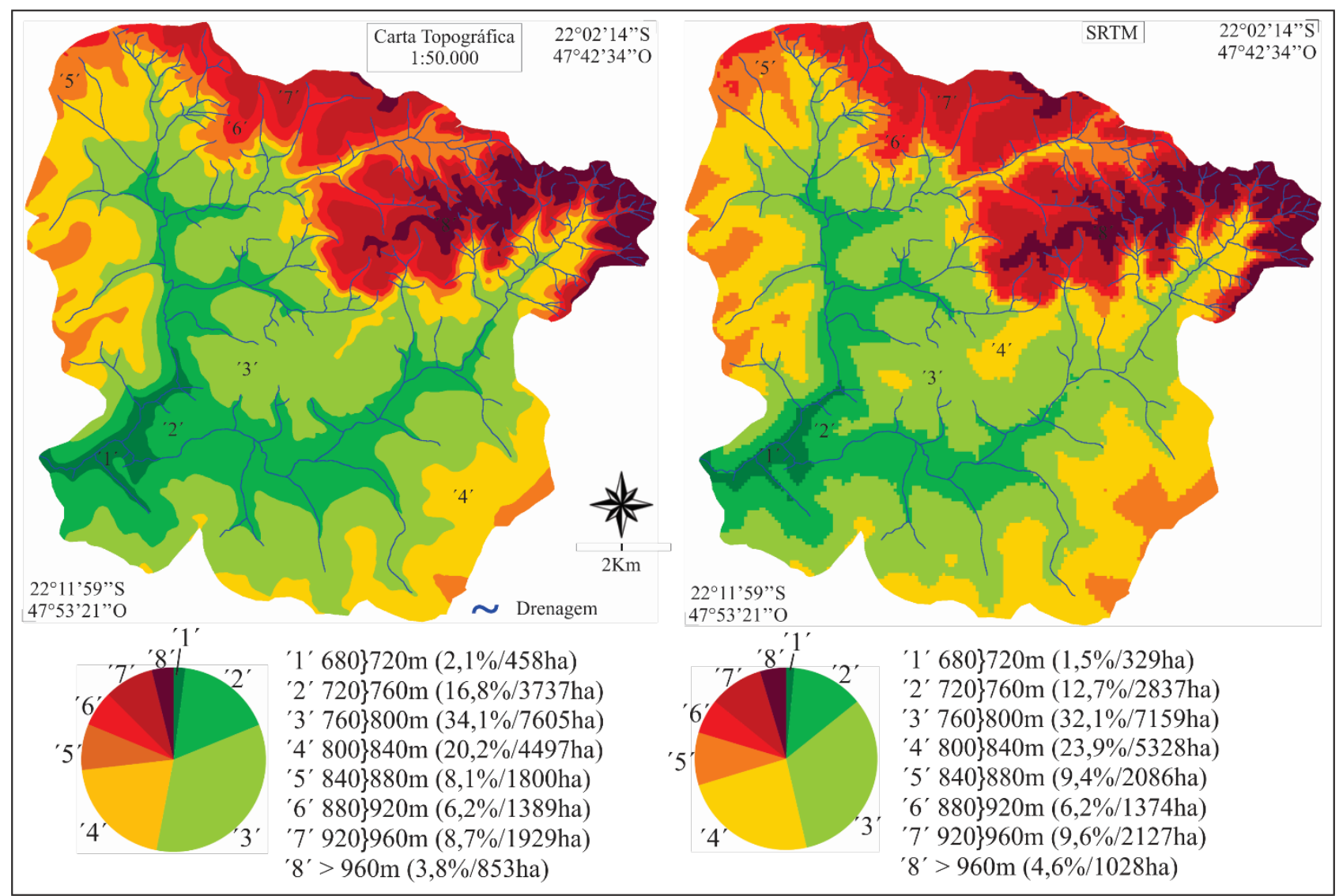

Figura 4 - Mapas hipsométricos.

No entanto, o índice KAPPA, encontrado para os MDTs, foi de 0.6594 que, de acordo com Landis \& Kock (1977) (Tabela 3), indica uma concordância substancial entre os dois mapas, revelando que o MDT SRTM pode ser fonte de dados hipsométricos com razoável confiança para bacias deste tamanho. Também, Tomas et al. (2014) verificou que a informação do MDT SRTM forneceu resultados aceitáveis em relação a uma referência com a mesma escala usada neste estudo.

Tabela 3 - Classes interpretativas do índice Kappa.

\begin{tabular}{c|c}
\hline Valor de Kappa & Qualidade do mapa \\
\hline$<\mathbf{0}$ & Pobre \\
\hline $\mathbf{0}-\mathbf{0 , 2}$ & Leve \\
\hline $\mathbf{0 , 2}-\mathbf{0 , 4}$ & Razoável \\
\hline $\mathbf{0 , 4 - 0 , 6}$ & Moderado \\
\hline $\mathbf{0 , 6}-\mathbf{0 , 8}$ & Substancial \\
\hline $\mathbf{0 , 8}-\mathbf{1 , 0}$ & Quase perfeito \\
\hline
\end{tabular}

Fonte: Landis e Kock, 1977.

Becek (2008) realizou um estudo de avaliação de erros de instrumentos do MDT SRTM sobre pistas de aeroportos, sem vegetação e em relevo plano, encontrando erro médio de 0 , o que significa que os erros estão relacionados com o relevo e com a vegetação presentes em outras áreas. O presente estudo foi realizado em uma bacia em que o relevo suave (entre 5 e 15\%) é interceptado por regiões de alta declividade, e são nessas áreas que ocorreram as maiores diferenças entre o MDT SRTM e o MDT referência.

Com relação à escala utilizada, Small \& Sohn (2015) correlacionaram escalas de MDTs e concluíram que, até a escala de 1:50.000 usada nesse estudo, o MDT SRTM apresentou boa correlação. 


\section{Fator LS}

Este fator é importante para estudos hidrológicos, pois calcula a distribuição espacial da capacidade de transporte de sedimentos pelo escoamento terrestre, entre as vertentes da bacia, de acordo com a convergência ou divergência do terreno (Moore \& Wilson, 1992).

Valores inferiores a 1 indicam áreas onde a deposição é maior do que o carreamento de sedimentos. Valores iguais a 1 participam de forma nula nos cálculos. Quando os valores são superiores a 1, eles são multiplicados ao modelo e resultam na ampliação do valor final, indicando maior participação do relevo na intensificação do processo erosivo (Silva \& Schulz, 2000).

Para o fator LS (Figura 5), o índice KAPPA foi 0.2584 que, segundo Landis \& Koch (1977) (Tabela 3), é interpretado como de baixa precisão. A discrepância geral (erros de omissão e de comissão) observada entre o LS/SRTM e o mapa referência foi de $43.93 \%$, significando que esta quantidade de pixels do mapa avaliado está alocada em classes diferentes do mapa referência.

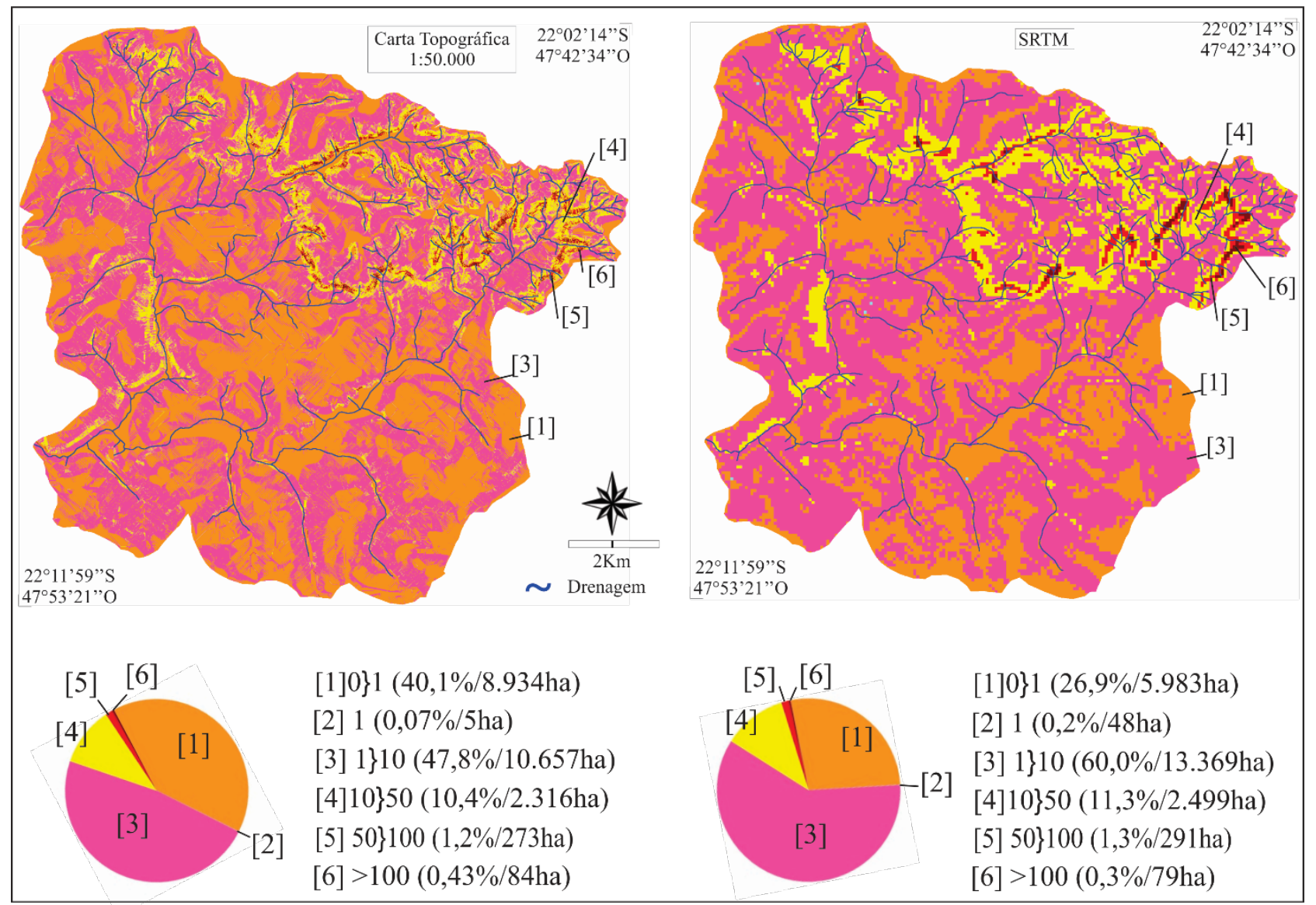

Figura 5 - Mapas Fator LS.

É importante ressaltar que esta análise trata dados gerados de forma totalmente independente (carta topográfica e MDT SRTM) e que, portanto, a coincidência entre os pixels é um forte indício de que a informação pode ser tratada como verdadeira.

Porém, a discrepância entre os pixels dos mapas não significa necessariamente que a informação seja irreal, pois o mapa referência não está livre de erros. Para tanto, é necessário analisar outras características dos mapas, como resolução espacial e metodologia de geração dos dados.

\section{Potencial Natural de Erosão}

O resultado obtido para potencial natural de erosão - PNE foi dividido em 4 classes, conforme indicado por Silva (2008). A matriz indicou que o mapa de perda natural gerado a partir do MDT SRTM - PNE SRTM apresentou erro em $50.27 \%$ dos pixels, o que mostra que mais da metade dos pixels estão presentes em classes diferentes dos encontrados no mapa referência. Percebe-se que a maior parte dos erros pode ser encontrada nas classes seguintes, mostrando que existe uma confusão entre as classes próximas. Tal fato pode ocorrer 
devido à arbitrariedade com que os limites entre as classes foram definidos, pequenas diferenças entre os dois mapas acabam por fazer pixels com valores próximos serem alocados em classes imediatas. $O$ índice KAPPA encontrado para perda natural foi de 0.2582. Esse valor coincide com o encontrado para o fator LS, revelando que os fatores $\mathrm{R} e$ $\mathrm{K}$ (que foram multiplicados ao MDT) não influenciaram as diferenças e semelhanças entre os mapas.

\section{Estimativa de Perda de Solo}

O resultado para a estimativa de perda de solo - EPS (Figura 6) está apresentado conforme escala de Irvem et al., (2007). Observou-se que para o mapa de EPS SRTM, 38.88\% da bacia apresentam muito baixa perda de solo, enquanto que no mapa referência, $45.32 \%$. Porém 29\% da área do EPS SRTM e 26\% no mapa referência apresentam perda de solo severa, muito severa e extremamente severa, o que mostra que o EPS SRTM superestimou os valores. Esse fato também foi observado por Sharma \& Tiwari (2014).

Percebe-se que as classes de maiores perdas são mais expressivas no EPS SRTM, em razão dos valores que eram menores que 1 no mapa
LS referência terem aparecido na classe entre 1 e 10 no LS SRTM.

A matriz de confusão mostrou que $43.38 \%$ dos pixels do EPS SRTM estão alocados em classes diferentes do mapa referência. Nesta matriz as classes com menores quantidades de pixels não levaram a maiores valores de erros, talvez por não se tratar de amplitudes das classes tão diferentes como no mapa do fator LS. O índice KAPPA obtido foi de 0.4199, maior do que do MDT. Esse valor pode ser interpretado como moderado, segundo Landis \& Koch (1977) (Tabela 3), indicando que o uso do MDT SRTM, para esta análise, forneceu resultados razoáveis.

A matriz de confusão mostrou que $43.38 \%$ dos pixels do EPS SRTM estão alocados em classes diferentes do mapa referência. Nesta matriz as classes com menores quantidades de pixels não levaram a maiores valores de erros, talvez por não se tratar de amplitudes das classes tão diferentes como no mapa do fator LS. O índice KAPPA obtido foi de 0.4199, maior do que do MDT. Esse valor pode ser interpretado como moderado, segundo Landis \& Koch (1977) (Tabela 3), indicando que o uso o MDT SRTM, para esta análise, forneceu resultados razoáveis.

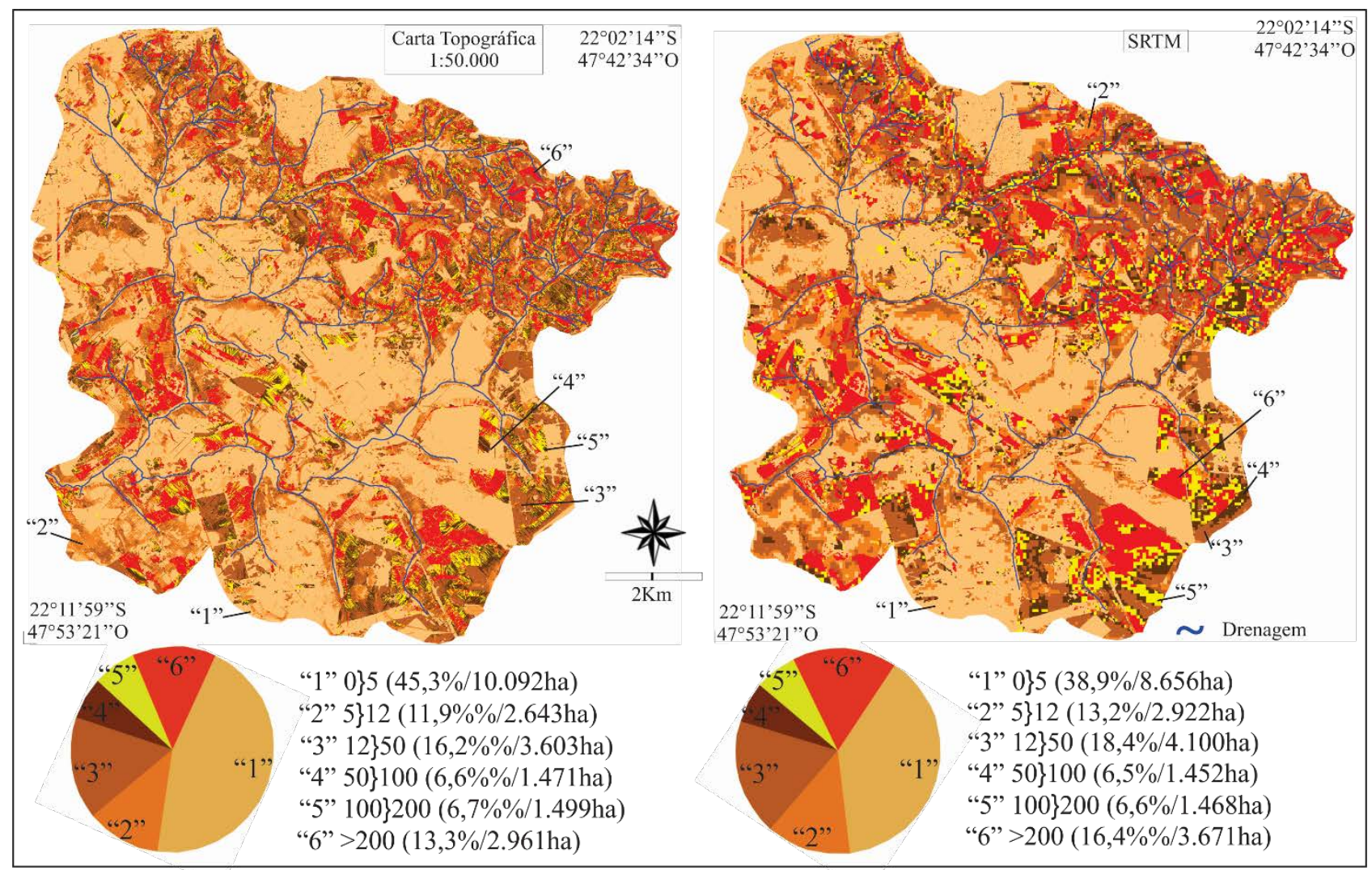

Figura 6 - Estimativa de Perda de Solo. 
O MDT é um de vários dados de entrada e seus erros podem ter sido diluídos pelo modelo, como observado em trabalhos anteriormente. Lin et al. (2013) utilizaram diferentes resoluções de MDT para calcular o carreamento de sedimentos em uma bacia de $81,7 \mathrm{Km}^{2}$ e encontraram resultados de diluição de erros pelo modelo. A alta resolução do mapa de uso e ocupação influenciou os mapas finais, no sentido de deixá-los similares. Rompaey \& Govers (2002) observaram que existe uma correlação entre a qualidade dos dados de entrada e a complexidade do modelo, notando que os resultados finais foram influenciados pelo modelo no sentido de mitigar erros dos dados de entrada. O resultado do modelo também foi satisfatório no estudo desenvolvido por Chaplot (2005), quando MDTs com resolução de $90 \mathrm{~m}$ foram utilizados para a estimativa de escoamento pelo modelo SWAT.

Kinsey-Henderson \& Wilkinson (2013) concluíram que em baixas declividades o MDT SRTM (90m) pode ser fonte de dados confiável. Neste estudo, onde a bacia é relativamente plana, o fator topográfico não influenciou as estimativas de perda de solo tanto quanto o mapa de uso e ocupação. Desta maneira, é possível concluir que a resolução grosseira do MDT SRTM, para esta bacia, não influenciou de forma a alterar em demasia o resultado para estimativa de perda de solo. Portanto, trata-se de uma fonte importante de dados onde não existem outras fontes de informação topográfica.

Para trabalhos cujo foco é o transporte de sedimentos alguns autores verificaram que a resolução do MDT SRTM é satisfatória, atingindo resultados satisfatórios quando comparados com outros modelos (de Vente et al., 2009, Lin et al., 2013, Tomas et al., 2014).

Alguns autores (Falorni et al., 2005; Guth, 2006), verificaram que o MDT SRTM apresenta muito ruído em áreas com declividade menor do que $5 \%$, ou seja, extremamente planas, localizadas nas planícies dos grandes rios e nas zonas costeiras. Porém, os mesmos autores afirmam ter conseguido informação precisa em outras regiões.

A morfologia é um fator com grande incidência sobre os erros encontrados nos MDTs (Aguilar, 2005), nesse estudo os erros em função da resolução grosseira do MDT SRTM foram amenizados pelo relevo suave presente na maior parte da bacia.

A variação dos resultados encontrados em avaliação de MDTs encontrada na literatura indica que a acurácia do MDT depende consideravelmente de parâmetros específicos de cada área, como características do terreno e tratamento anterior dos dados (Athmania \& Achour, 2014). Portanto, estudos que verificam a qualidade de dados de entrada, sob condições adversas, são importantes para que a confiabilidade dos resultados dos modelos seja satisfatória.

\section{CONCLUSÃO}

A maior parte dos erros foi detectada ao longo dos limites entre as classes dos mapas que foram comparados. Esse fato pode ser percebido na matriz de erro do mapa hipsométrico, onde todos os erros aconteceram entre as classes subsequentes. Nas outras matrizes (mapas do fator LS, mapas PNE e EPS) isso também ocorreu, porém com maiores ocorrências em classes mais distantes.

Para escolher qual resolução e procedência dos dados de entrada são mais apropriadas é necessário observar características específicas da origem dos dados disponíveis e da região a ser estudada. Os resultados desta pesquisa indicaram que em bacias cujos fatores LS não são os mais relevantes para o resultado de estimativa de perda de solo, a resolução grosseira do MDT SRTM pode ser amenizada pela qualidade dos dados de uso e ocupação da terra, mesmo em bacias pequenas, como a estudada.

Com isso conclui-se que o MDT SRTM é uma fonte importante de dados de entrada, porém a influência de sua resolução sobre a aplicação do modelo deve ser analisada para que não prejudique a qualidade dos resultados.

\section{AGRADECIMENTOS}

Os autores são gratos a Coordenação de Aperfeiçoamento de Pessoal de Nível Superior - CAPES pela bolsa de estudos concedida. 


\section{REFERÊNCIAS}

AGUILAR, J.; AGUERA, F.; AGUILAR, M.A.; CARVAJAL, F. Effects of terrain morphology, sampling density, and interpolation methods on grid DEM accuracy. Photogrammetric Engineering and Remote Sensing, v. 71, n. 7, p. 805-816, 2005

ATHMANIA, D.; ACHOUR, H.. External Validation of the ASTER GDEM2, GMTED2010 and CGIAR-CSI- SRTM v4.1 Free Access Digital Elevation Models (DEMs) in Tunisia and Algeria. Remote Sensing, v. 6, p. 4600-4620, 2014.

BECEK, K. Investigating error structure of shuttle radar topography mission elevation data product. Geophysical Research Letters, v. 35, L15403, 2008.

CHAPLOT, V. Impacto f DEM mesh size and soil map scale on SWAT runoff, sediment, and NO3-N loads predictions. Journal of Hydrology, v. 312, p. 207-222, 2005.

CHAVES, H.M.L. Estimativa da erosão atual e potencial no Vale do São Francisco. Relatório final de Consultoria. CODEVASF/FAO, Brasília, 1994.

COHEN, J. A coefficient of agreement of nominal scales. Educational and Psychological Measurement, v. 20, p. 3746, 1960.

CONGALTON, R.G.A review of assessing the accuracy of classifications of remotely sensed data. Remote Sensing of Environment, v. 37, p. 35-46, 1991.

CONGALTON, R.G. \& Green, K. A practical look at sources of confusion in error matrix generation. Photogrammetric Engineering and Remote Sensing, v. 59, n. 5, p. 641-644, 1993.

CUNHA, R. C.; DUPAS, F. A.; PONS, N. A. D.; TUNDISI, J. G. Análise da influência das variáveis ambientais utilizando inferência Fuzzy e zoneamento das vulnerabilidades. Estudo do caso da bacia hidrográfica do ribeirão do Feijão, São Carlos, SP. Revista Geociências, v. 30, n. 3, p. 399-414, 2011.

DESMET, P.J.J. \& GOVERS, G. A GIS-procedure for automatically calculating the USLE LS factor on topographically complex landscape units. Journal of Soil and Water Conservation, v. 51, n. 5, p. 427-433, 1996.

FALORNI, G.; TELES, V.; VIVONI, E.R.; BRAS, R.L.; AMARATUNGA, K.S. Analysis and characterization of the vertical accuracy of digital elevation models from the Shuttle Radar Topography Mission. Journal of Geophysical Research. v. 110, n. F2, p. 1-20, 2005.

FARINASSO, M.; CARVALHO J.R.; O.A.; GUIMARÃES, R.F.; GOMES, R.A.T.; RAMOS, V.M. Avaliação Qualitativa do Potencial de Erosão Laminar em Grandes Áreas Por Meio Da EUPS - Equação Universal de Perdas de Solos Utilizando Novas Metodologias em S.I.G. para os Cálculos dos seus Fatores na Região do Alto Parnaíba - PiMa. Revista Brasileira de Geomorfologia, v. 7, n. 2, p. 7385, 2006.

FLUET-CHOUINARD, E.; LEHNER, B.; REBELO L.M.; PAPA, F.; HAMILTON, S.K. Development of a global inundation map at high spatial resolution from topographic downscaling of coarse-scale remote sensing data. Remote Sensing of Environment, v. 158, p. 348-361, 2015.

FOODY, G.M. Classification accuracy comparison: Hypothesis tests and the use of confidence intervals en evaluations of difference, equivalence and non-inferiority. Remote Sensing of Environment, v. 113, p. 1658-1663, 2009.

GERICKE, A. Soil loss estimation and empirical relationships for sediment delivery ratios of European river catchments. International Journal of River Basin Management, v. 13, p. 179-202, 2015

GUTH, P. Geomorphometry from SRTM: comparison to NED. Photogrammetric Engineering \& Remote Sensing, v. 72, n. 3, p. 269-277, 2006.

IBGE - Instituto Brasileiro de Geografia e Estatística. Folhas topográficas de São Carlos - SF-23-Y-A-I-1 e Corumbataí - SF-23-Y-A-2. Escala 1:50.000, 1972.
IRVEM, A.; TOPALOGLU, F.; UYGUR, V. Estimating Spatial Distribution of Soil Loss Over Seyhan River Basin in Turkey. Journal of Hydrology, v. 336, p. 30-37, 2007.

KINSEY-HENDERSON, A. E.; WILKINSON, S. N. Evaluating Shuttle radar and interpolated Ds for slope gradient and soil erosion estimation in low relief terrain. Environmental Modelliing and Software, v. 40, p. 128-139, 2013.

KORADA, H. V. D. R.; RAO, V. Soil erosion assessment in Rangit catchment, India through a process-based model in the geospatial environment. Geocarto International, v. 29, p. 207-219, 2014.

LANDIS J. R.; KOCK G. G. The measurement of observer agreement for categorical data. Biometrics, v. 33, p. 159-74, 1977.

LI, P.; SHI, C.; LI, Z.; MULLER, J.P.; DRUMMOND, J.; LI, X.; LI, T.; LI, Y.; LIU, J. Evaluation of ASTER GDEM using GPS benchmarks and SRTM in China. International Journal of Remote Sensing, v. 34, p. 1744-1771, 2013.

LIN, S.; JING, C.; COLES, N. A.; CHAPLOT, V.; MOORE, N. J.; WU, J. Evaluating DEM source and resolution uncertainties in the soil and water assessment tool. Stochastic Environmental Research and Risk Assessment, v. 27, p. 209-221, 2013.

MELLO, C. R.; SÁ, M. A. C.; CURI, N.; MELLO, J. M.; VIOLA, M. R.; SILVA, A. M. Erosividade mensal e anual da chuva no Estado de Minas Gerais. Pesquisa Agropecuária Brasileira, v. 42, n. 4, p. 537-545, 2007.

MIRANDA, E. E. (Coord.). Brasil em Relevo. Embrapa Monitoramento por Satélite, 2005. Disponível em: $<$ http://www.relevobr.cnpm.embrapa.br>. Acesso em: 26 jun. 2011.

MOORE, I.D.; WILSON, J.P. Length-slope factors for the revised universal soil loss equation: simplified method of estimation. Journal of Soil and Water Conservation, v. 47, n. 5, p. 423-428, 1992.

MORADI, A.; METIVIER, L.; VIRON, O.; CALMANT, S.; MERING, C. Evaluation of MODIS data for improved monitoring of the Caspian Sea. International Journal of Remote Sensing, v. 35, p. 6060-6075, 2014.

MOREIRA, M. C.; CECÍlIO, R. A.; PINTO, F. A. C.; LOMBARDI NETO, F; PRUSKI, F. F. Programa Computacional para Estimativa da Erosividade da Chuva no Estado de São Paulo Utilizando Redes Neurais Artificiais. Engenharia na Agricultura, v 14, n. 2, p. 88-92, 2006.

NISHIYAMA, L. Mapeamento Geotécnico preliminar da quadrícula de São Carlos, SP. Tese de Doutorado em Geotecnia, Escola de Engenharia de São Carlos, Universidade de São Paulo, 1991.

OLIVEIRA, J. B.; PRADO, H. Levantamento pedológico semidetalhado do estado de São Paulo: quadrícula de São Carlos. Instituto Agronômico de Campinas, 188p., 1984.

OLIVEIRA, P. T. S.; SOBRINHO, A. T.; RODRIGUES D. B. B.; PANACHUKI, E. Erosion Risk Mapping Applied to Environmental Zoning. Water Resource Management, v. 25, p. 1021-1036, 2011.

PANDEY, A.; CHOWDARY, V. M.; MAL, B. C. Identification of Critical Erosion Prone Areas in the Small Agricultural Watershed Using USLE, GIS and Remote Sensing. Water Resources Management, v. 21, p 729-746, 2007.

PIMENTA, M. T. Diretrizes para a aplicação da equação universal de perdas dos solos em SIG: factor de cultura $\mathrm{C}$ e factor de erodibilidade do solo K. INAG/DSRH Portugal, 1998.

RABUS, B.; EINEDER, M.; ROTH, A.; BAMLER, R. The shuttle radar topography mission-a new class of digital elevation models acquired by spaceborne radar. Journal of Photogrammetry and Remote Sensing, v. 57, n. 4, p. 241262, 2003. 
REXER, M.; HIRT, C. Comparison of free high-resolution digital elevation data sets (ASTER 1 GDEM2, SRTM v2.1/v4.1) and validation against accurate heights from the Australian National 2 Gravity Database. Australian Journal of Earth Sciences, v. 61, n. 2, p. 213-226, 2014.

RODRIGUEZ, E.; MORRIS, C.S.; BELZ, J.E. A global assessment of the SRTM performance. Photogrammetric Engineering \& Remote Sensing, v. 72, p. 249-260, 2006.

ROMPAEY, A. J. J.; GOVERS, G. Data quality and model complexity for regional scale soil erosion prediction. International Journal of Geographical Information Science, v.16, n. 7, p. 663-680, 2002.

SHAN, X.; TANG, P.; HU, C. An automatic geometric precision correction system based on hierarchical registration for $\mathrm{HJ}-1 \mathrm{~A} / \mathrm{B}$ CCD images. International Journal of Remote Sensing, v. 35, p. 7154-7178, 2014.

SMALL, C.; SOHN, R. Correlation scales of digital elevation models in developed coastal environments. Remote Sensing of Environment, v. 159, p. 80-85, 2015.

SHARMA, A.; TIWARI, K.N. A comparative appraisal of hydrological behavior of SRTM DEM at catchment level. Journal of Hydrology, v. 519, p. 1394-1404, 2014.

SILVA, A. M. Potencial Natural de Erosão no município de Sorocaba, São Paulo, Brasil. Revista Internacional de Desastres Naturales, Accidentes e Infraestructura Civil, v. 8, n. 1, p. 5-14, 2008.

SILVA, A. M.; ALVARES, C. A. Levantamento de informações e estruturação de um banco de dados sobre a erodibilidade de classes de solos no estado de São Paulo. Revista Geociências, v. 24, n. 1, p. 33-41, 2005.

SILVA, A. M.; SCHULZ, H. E. Estimativa e análise do fator topográfico (LS) da Equação Universal de Perda de Solo com o uso de aplicativo informatizado compatível com software de geoprocessamento. Revista FatorGIS, 2000.

SILVA, V. C. Estimativa da erosão atual da bacia do Rio Paracatu (MG/GO/DF). Pesquisa Agropecuária Tropical, v. 34, n. 3, p. 147-159, 2004.

THOMAS, J.; JOSEPH, S.; THRIVIKRAMJI, K.P.; ARUNKUMAR, K.S. Sensitivity of digital elevation models: the scenario from two tropical mountain river basins of the Western Ghats, India. Geosciences Frontiers, v.5, p. 893909, 2014.
TUNDISI J. G.; TUNDISI, T. M.; DUPAS, F.A.; SILVA E SOUZA, A. T. Uso atual e uso potencial do solo no município de São Carlos, SP - base do planejamento urbano e rural. Relatório final de Projeto de Políticas Públicas da FAPESP, proc. 98/10924-3, 2007.

VALERIANO, M. M.; ROSSETTI, D. F. Topographic modeling of Marajó island with SRTM data. Revista Brasileira de Geomorfologia, v. 9, n.1, p. 53-64, 2008.

DE VENTE, J.; POESEN, J.; GOVERS, G.; BOIX-FAYOS, C. The implications of data selection for regional erosion and sediment yield modeling. Earth Surface Processes and Landforms, v 34, p 1994-2007, 2009.

WEILL, M. A. M.; SPAROVEK, G. Estudo da Erosão na Microbacia do Ceveiro (Piracicaba, SP). I - Estimativa das Taxas de Perda de Solo e Estudo de Sensibilidade dos Fatores do Modelo EUPS. Revista Brasileira de Ciência Solo, v. 32, p 801-814, 2008.

WISCHMEIER, W. H., SMITH, D. D. Predicting Rainfall Erosion Losses: a Guide to Conservation Planning. United States Department of Agriculture, Agriculture Handbook, 58p., dez, 1978.

YANG, L.; MENG, X.; ZHANG, X. SRTM DEM and its application advances. International Journal of Remote Sensing. v. 32, n.14, p. 3875-3896, 2011.

ZHANG, Y; DEGROOTE, J; WOLTER, C.; SUGUMARAN, R. Integration of Modified Universal Soil Loss Equation (MUSLE) Into a Gis Framework to Assess Soil Erosion Risk. Land Degradation \& Development, v. 20, p. 84-91, 2009.

ZUQUETTE, L. V. Mapeamento Geotécnico preliminar na região de São Carlos. Dissertação de Mestrado em Geotecnia, Escola de Engenharia de São Carlos, Universidade de São Paulo, São Carlos, 1981. 